\title{
Building avatar armies with fish and flies
}

\author{
Personalizing zebrafish and fly models may help determine the best course of treatment for cancer patients.
}

\author{
Ellen P. Neff
}

W

hen a patient arrives at a

hospital in need of antibiotics for an infection, they will often receive an antibiogram. Samples will be tested to check which available treatment should be best, in terms of both fighting the patient's infection and avoiding resistance mechanisms.

It's a simple check that's been around for decades, says Rita Fior, a developmental-turned-cancer biologist at the Champalimaud Centre for the Unknown in Lisbon. Things are, unfortunately, not so simple for cancer.

Clinical trials inform therapy guidelines for various cancers and ever improving biomarkers can, in some cases, narrow down the available options. But not always. Often, an individual's particular therapy can come down to chance. One clinic may use drug A; another, Drug B...

"To me, this was a bit shocking that this still happens nowadays," says Fior. "We can go to Mars, but if you have cancer you are still choosing the best chemo[therapy] by chance."

The emergence of personalized medicine-choosing the right treatment for each individual patient-might shift the odds. The technologies to profile individual tumors and sequence patient genomes for different mutations and biomarkers that could indicate treatment efficiency are improving and becoming less expensive. Ultimately though, a treatment must still be chosen. "You need to actually test the patient cells in a personalized way to see if the cells respond to Drug A or Drug B," says Fior.

In preclinical cancer research, one of the gold standards for testing therapies is a patient-derived xenograft (PDX) mouse model. Primary samples are taken from individual patients and transplanted across species into immunodeficient mice, yielding personalized 'avatars' that can be used to test the efficacy and potential toxicity of a particular treatment. Researchers are currently working through some growing pains while creating increasingly sophisticated PDX mouse models, but generating mice still takes time-time patients may or may not have.

What can take months to achieve in a mouse can, it turns out, be pulled off in just a few days, in greater numbers and at less

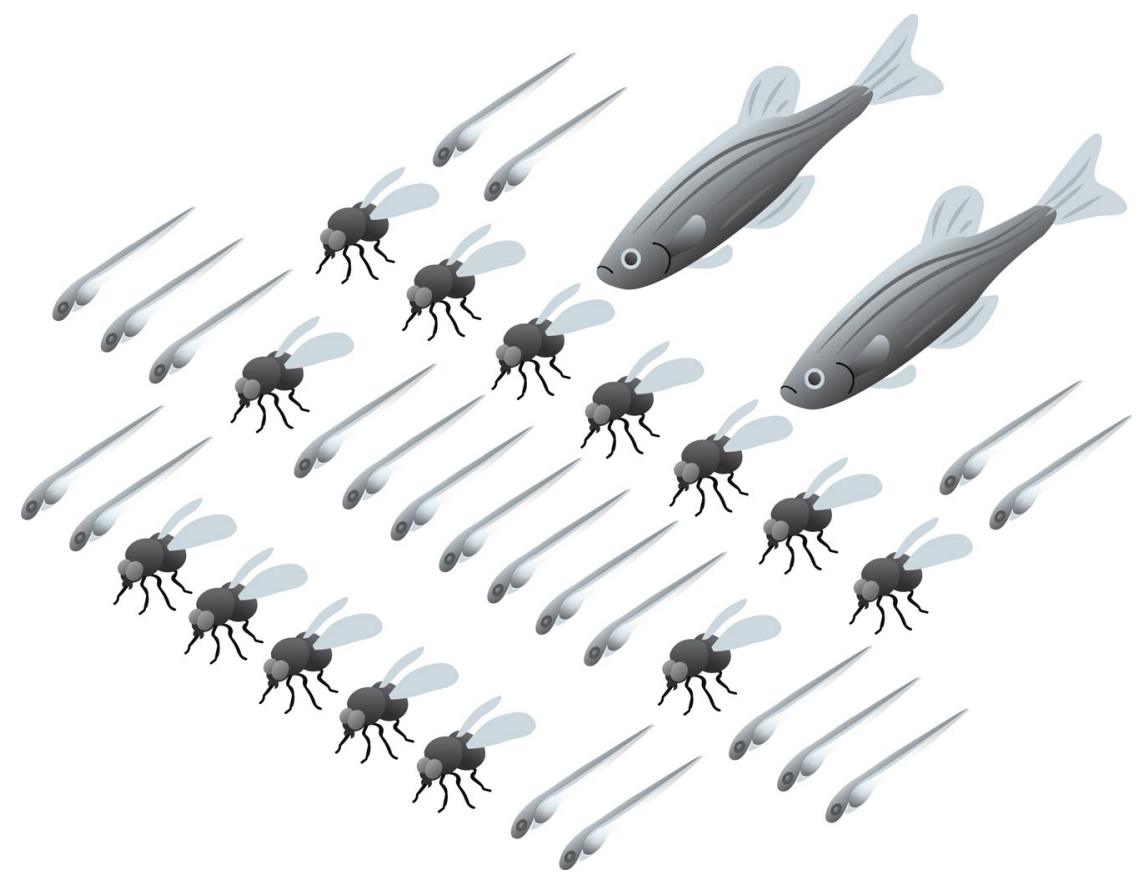

Called for duty | Zebrafish and fruit flies can be made quickly and in large numbers. With technologies that enable the animals to be personalized to a given cancer patient, avatar armies may be rising in the future. Credit: E. Dewalt, Springer Nature

cost, in smaller animals. Personalization has been coming to fish and flies too, and 'avatar armies' of such animals may soon help in the fight against cancer.

\section{Release the larvae}

When cell biologist Graham Dellaire decided it was time to make the move into animals, he went with the zebrafish, Danio rerio. Oncologist Jason Berman had recently brought his fish models to Dalhousie University, and the two struck up a collaboration to create a zebrafish model of acute promyelocytic leukemia (APL), a rare blood cancer.

The plan was to engineer a zebrafish with mutated PML genes, a contributing factor to APL in humans. But they quickly swam into a problem: zebrafish don't possess a PML paralog. Somewhere along their evolutionary history, they lost the equivalent gene to time.

Undeterred, they looked to a different approach. Rather than try to create a mutation that would cause cancer in the fish, why not just add the cancer directly?
Zebrafish xenografts were emerging at the time, with human melanoma cell lines successfully engrafted into larval fish ${ }^{1}$. For Berman and Dellaire, it worked for human leukemia cells lines too ${ }^{2}$.

"Not only did the cells engraft and grow in the zebrafish larvae, but they did so in such a rapid time frame that you could actually test drug responses by adding them to the water in the span of days-this was completely not feasible in a mouse model," says Berman, now the CEO and scientific director at the Children's Hospital of Eastern Ontario Research Institute. "That's what gave us this idea: could this approach be used for personalized therapy?"

In work published in 2015, Berman, Dellaire, and collaborators injected human cancer cell lines as well as primary tumors from two children with T-Cell acute lymphoblastic leukemia into the larval yolk sac and then screened a number of drugs targeting different cancer-causing pathways. In the fish, NOTCH1-mutations in which are found in a number of human 


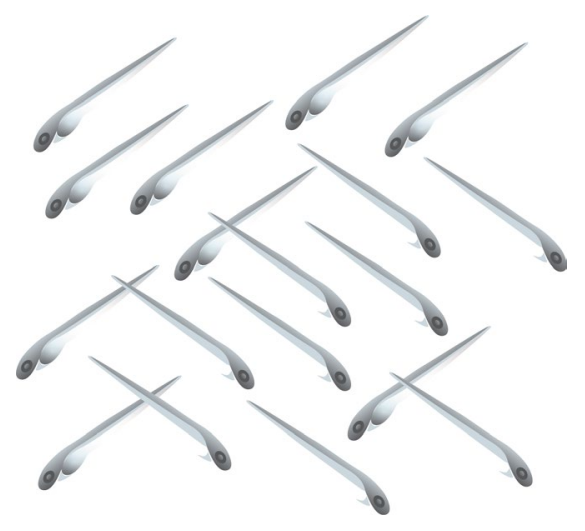

Credit: E. Dewalt, Springer Nature

cancers-was implicated. They had no advanced genetic information about the two patients, but subsequent exome sequencing revealed that a NOTCH1 mutation was indeed present in one of the children ${ }^{3}$.

From the phenotypic data in the fish, the team was able to find a biological answer in the span of just a week. "In that case, Patient 1 could have benefited from adding a NOTCH inhibitor to their treatment, whereas Patient 2 would not have-they might have just had side effects without benefit," says Berman. Both patients were at the time responding to standard therapies, but Berman and Dellaire had proof-of-concept that personalizing the fish held promise.

Over the past few years, other labs have started working with primary tumors, including for other leukemias, pancreatic, prostate, and breast cancers, and even neuroendocrine tumors (reviewed in ref. ${ }^{4}$ ). Fior has had success in generating zebrafish PDX (zPDX) models of solid tumors; the first was for colorectal cancer ${ }^{5}$, and her lab in Portugal has recently started developing larval zebrafish models from breast cancer micro-biopsies. Beyond testing drugs, they've also shown that the zPDXs will respond to radiotherapy in a way that matches the patients from which the tumors were sampled ${ }^{6}$.

The larvae bring a number of advantages. Whereas mice must have components of their immune system knocked out so that the transplanted human cells won't be rejected, zebrafish hatch sans adaptive immunity-for their first few weeks of life, they have no B, $\mathrm{T}$, or Natural Killer cells to interfere with foreign material. The larvae are naturally transparent-although many working with xenografts make use of the ghostly white casper strain ${ }^{7}$, developed by Richard White to lack any pigmentation whatsoever. Zebrafish also produce a lot of larvae-with a skilled technician doing the tumor cell injections, researchers can make hundreds of fish at a time. Larval numbers can reasonably reach into the hundreds-though in the case of primary tumors, that can be limited somewhat by the amount of biopsy material available. This provides an opportunity for scale that is an ethical and expense challenge to undertake in mouse, says Dellaire. And it's easy to add drugs, alone or in different combinations, to the water for quick read outs. "The fish is great to look at the efficacy of drugs," says Berman. "And one of the advantages to putting this into a whole living organism like larvae as opposed to just testing it a petri dish is that we can see side effects." Is, for example, development affected? That's particularly important for pediatric cancers, says Berman.

But xenografting human tumors into any animal comes with challenges, such as keeping track of which cells belong to a human and which to the fish. Injected cells can be fluorescently labeled, but whereas cell lines can be tagged permanently, any dyes added to primary tumors will eventually fade as the cells divide, notes Berman.

Under the microscope, human and fish cells do look distinct says Fior, and researchers can use human-specific antibody markers, such as that which recognizes human HLA, that don't cross-react with fish cells to help confirm cellular identities.

To assess cancer proliferation and response to treatment, Dellaire \& Berman developed an ex vivo assay to quantify their results: the larvae are dissociated and the fluorescently labeled human cells counted. Fior has been taking advantage of the optical transparency of the larvae to observe details such as cell death, angiogenesis, and the emergence micrometastases under the microscope. "Every fish goes to the confocal," she says, for single-cell quantification. "It's not so high throughput in that sense, but I prefer to be sure about the results."

Growing up and becoming human(ized) Whole animals come with context that's inherently missing in vitro. "We are able to see the tumor microenvironment," says Fior, "The innate macrophages, neutrophils, and so on." In a PDX model, each tumor comes with its patient's unique genetics and will reconstitute a different microenvironment, with different impacts on tumorigenesis and different responses to treatments. "We can see that some [human] tumor cells are able to suppress the innate immunity of the fish, or are permissive and then cleared, "she says.

But larvae do have limits. Although the field has upped the water temperature for larval zebrafish from $28^{\circ} \mathrm{C}$ to $35^{\circ} \mathrm{C}$, that's still about 2 degrees lower than the temperature a tumor will find in the human body. And eventually, adaptive immunity does kick in to kick out the human cells. That can limit the overall length of tumor progression and metastases studies, says David Langenau of the Massachusetts General Hospital and Harvard Medical School. Plus, the small size of the larvae does limit the number of human cells that can be engrafted and makes it more difficult to gavage precise amounts of a drug relative to larger animals.

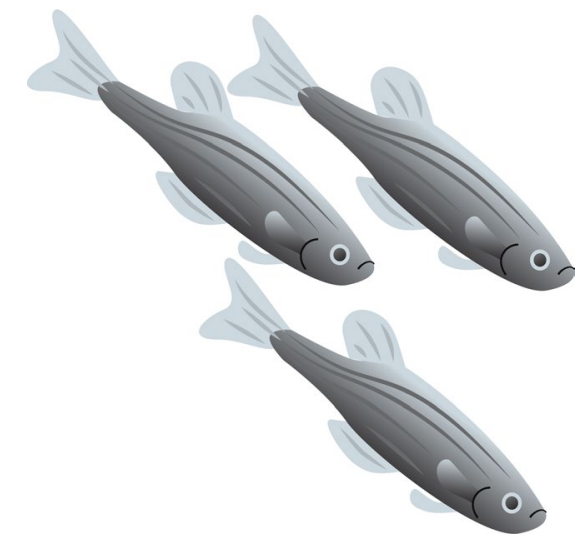

Credit: E. Dewalt, Springer Nature

Langenau has long worked with various zebrafish cancer models. But he and his lab recently decided to try and go big, with the establishment of an adult zebrafish xenograft model. As with mice, the zebrafish had to be made immunocompromised to prevent rejection of the tumors, but the genome engineering technology to do so was readily available: the resulting prkdc-/-, il2rga-/casper adults lack T, B, and NK immune cells. The bigger challenge was figuring out the husbandry conditions needed to raise their water temperature to meet that of the human body and to optimize fish growth so they did not develop opportunistic infections. Slow acclimation, a new diet, and antibiotics did the trick.

In a recent work published in Cell, the lab transplanted several cancer cells lines as well as primary tumors from children with muscle-afflicting rhabdomyosarcoma ${ }^{8}$. The latter were injected into the eye musculature of the adult models, enabling single-cell resolution observations of tumor behavior using standard confocal microscopy as the team tested different combination therapies.

For further validation, they also tested several mouse PDX models-and the fish rose to the occasion. The data suggests that human tumors grow and respond to drugs in the same way, whether they are grown in an immunodeficient fish or mouse, says Langenau.

Another hurdle, whether for larvae or adult zebrafish and for mice too, will be to 'humanize' the animals. "There are some big caveats with both mice and zebrafish 
for human xenograft studies, and the most important one is probably the mismatch between growth factors and cytokines expressed by these animals," says Dellaire. Giving animals different elements of the human immune system, in addition to cancerous cells, has been underway for mice, and such work is in progress for zebrafish too.

"People recognize that the behavior of a cancer cell is not just dependent on the cancer cell itself, but things that are going on its surrounding environment," says Berman. He and his lab have been working on 'humanizing' larvae - in work published late last year, they developed transgenic casper fish that express three different human cytokines that enhance human hematopoietic stem cell survival "We've been able to show that when you put patient-derived cancer cells into those fish, [the cells] grow better," says Berman.

\section{Mirroring mutations}

Engrafting cells from a patient's tumor into an animal brings that patient's mutations along with it, but even with partial humanization there are still immune components missing in a PDX model. Another approach labs might take is to genetically manipulate animals to induce endogenous tumors, naturally arising within the context of immunocompetent animals. That too is becoming increasingly personalized, with animals created with genetic mutations that mirror those found in patients. Patient profiles, however, can be complex-people will carry various oncogenes and tumor suppressor genes, along with numerous 'passengers' that are unique to each individual but may or may not impact the development or progression of their cancer. The question has become, what mutations matter, and how many?

"We're really good at rescuing a preclinical model," says Ross Cagan at the Icahn School of Medicine at Mount Sinai in New York. "But it rarely translates into people." Rather than making a genetic model with a single 'hit'-animals with just one oncogene or tumor suppressor expressed or removed-his lab has been layering them on. Not in the mouse though-in the fruit fly, Drosophila melanogaster.

Though the fly has long been used as a developmental model, its role in cancer research does go pretty far back too-Mary Stark first observed tumors in the animals in $1919^{10}$. As more and more conserved genes and signaling pathways have been observed between the fly and humans, its role as a disease model has been expanding and now includes models of different cancers. Transgenesis in flies allows researchers to overexpress and/or knockdown different

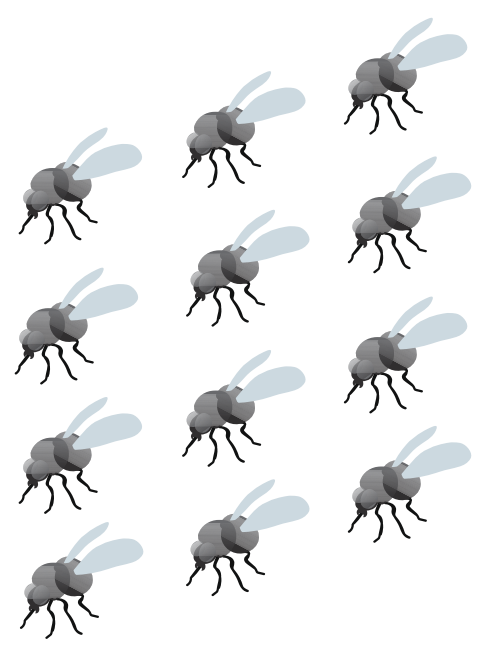

Credit: E. Dewalt, Springer Nature

genes, and with the Gal4/UAS system and a large and growing catalogue of promotors, gene expression can be carefully controlled within specific tissues ${ }^{11}$.

The first cancer model Cagan worked on was for a medullary thyroid cancer-flies don't have a thyroid, but they do have a paralogous gene for one commonly mutated in the disease. Cancer researchers knew this cancer was primarily driven by mutations in the RET oncogene, says Cagan, but at the time-the late 90s-they didn't know what to do with that information. "Like all good fly biologists, I said that's a signaling problem," he recalls. "You should just study that in fruit flies." The colleague he was chatting with, cancer biologist Paul Goodfellow, was skeptical but sent a postdoc to Cagan's lab nonetheless.

They built a fly model that overexpressed RET in the developing fly retina to cancerous effect. Treating that model with a compound called ZD6474 proved therapeutic in the fly ${ }^{12}$ - and eventually in humans with medullary thyroid cancer, for whom it is marketed as Vandetanib. "Going through that process and meeting some of the patients taking [Vandetanib] ...that was really motivating for me," says Cagan. "That's when I began to shift my lab from development to disease."

Despite that success story-and just as in mice-there has been a gulf between success in the fly and success in patients. Erdem Bangi, a former postdoc in Cagan's lab, decided to investigate the disconnect. Focusing on colorectal cancer, he took 16 drugs that had made it into clinical trials and gave them to a fly with a single cancerous hit. "They worked beautifully," says Cagan. In the clinic however, all 16 had failed.

So Bangi, now a professor at Florida State University, increased the hits from one to four, creating multigenic fly models containing various combinations of four cancer-related genes: ras, $p 53$, pten, and apc. The drugs failed in the flies then $t^{13}{ }^{13}$. In 2019, the lab upped the complexity even more, publishing a 9-hit fly based on the mutation profile found in a KRAS-mutant metastatic colorectal patient who was not responding to therapy; in that model, they identified a combination therapy that stabilized the patient for over a year ${ }^{14}$. In the lab, Cagan says they've since made it up to 18 hits.

To create such complex genetic models, long strands of nucleic acids are engineered with multiple hairpins; each hairpin correspond to particular gene to be targeted for overexpression, such as different tumor suppressors or oncogenes The multi-hairpin oligo is placed under an inducible, tissuespecific promotor, such that those genes will only be expressed in a particular place. The resulting transgenic flies are then crossed with others carrying RNAi constructs to knockdown expression of genes to be removed from the mix, thus creating stable lines of flies with a particular combination of over- and under-expressed genes.

Complexity is also on the rise in transgenic zebrafish as well. Using a system called miniCooper ${ }^{15}$, transgenes can be ferried via a plasmid injected into developing embryos underneath the mitfa promotor, which controls the expression of the pigmented melanocytes that give the zebrafish its characteristic stripes. Add it to what would otherwise be pigmentless casper fish and the stripes are restored-and any additional genetic cargo delivered.

In the first miniCooper publication, researchers expressed the BRAF oncogene along with SETDB1 in fish melanocytes, showing that the combination accelerated tumors. Adding more genes to the mix might be as simple as adding more plasmids. Richard White's lab at Memorial Sloan Kettering Cancer Center in New York is working on just that, expanding on the miniCooper system with the development of their "One Shot" method. The project has had a personalized element from the start, says $\mathrm{MD} / \mathrm{PhD}$ student Joshua Weiss.

In 2017 , genomic analysis ${ }^{16}$ indicated that the mutation profile of acral melanoma, a rare form of skin cancer found on palms and the bottom of feet, is considerably different from that of the more common cutaneous form; the exact genetics drivers, however, are still being worked out, says Weiss. Rather than guess what might be important, they looked to an acral melanoma patient whose tumors had been sequenced as part of a clinical trial. Weiss has focused in on four genes, injecting plasmids containing each, along with a GFP tag, into hundreds 
of zebrafish embryos. The study is still in progress, but preliminary results suggest the patient-mirroring mutations lead to tumors differentially appearing on extremities-in the fish's case, on its fins.

The trick to introduce so many transgenes into a fish has been a simple one, says Weiss: just include a little less DNA per plasmid to reduce the risk of toxicity. They've made it up to 8 genes in a single animal-a few shy of fly, but still more complexity and in a simpler, faster way than has been possible in rodents.

These larvae have been proving fruitful for studying primary tumors that form in the fish, but the tumors can appear everywhere-how then to tell the difference between a primary tumor and secondary metastases? Here, a new technique in adult fish might help. White's lab recently developed a method to target transgenes to specific places on the body called Transgene Electroporation in Adult Zebrafish-or TEAZ ${ }^{17}$. The DNA constructs carry oncogenes, suppressors, or even CRISPR/Cas 9 cassettes to create specific mutations that can be placed as desired in immunocompetent adults. "It's a way to test more directly the role of position and the microenvironment of a cancer," says Weiss.

\section{Avatars assemble}

Whether fish or fly, the goal of personalized avatars is ultimately to help the patients they are intended to model. "The motivation is really to help direct treatment, to choose the right treatment for each individual patient," says Fior.

Validation of the larval zPDX models is underway to confirm how predictive the animals really are. "We treat our fish with the same drug as the patients are taking and compare the results," says Fior. So far, 18 out of 21 of her colorectal avatars have mirrored their patients, and the lab is working to increase the numbers for other cancers as well.

Likewise with the larvae in Canada. "Currently, we're trying to show some preclinical response," says Berman. "We're involved in some projects now looking retrospectively at responses in mice and responses in patients." Such fish may soon be part of a large effort in Canada called PROFYLE-Precision oncology for young people-that hopes to find treatments for children with cancers that have recurred or are refractory to common standard therapies. "Normally, we wouldn't have any options," says Berman. With the larval models, the goal is to take a piece of the child's tumor into the fish to test in real time its drug responses to different treatments. "Just by screening a number of drugs in the zebrafish

\section{Box 1 | Where do the mice go?}

Where then do these fish and fly developments leave mice? At the end of the day the mammals are still closer proxies to humans, in terms of their anatomy and physiological details such as their immune system. But their role may change if other avatars continue to progress.

"For personalized medicine-for actually advising therapy, I don't think you need the mice," says Fior. They can just take too long-following diagnosis, doctors may only have a few weeks to decide on the course of treatment. "This is not enough time for mouse." A PDX mouse would still be worthwhile to have ready though for the future, in the event that the initial treatment is unsuccessful or if tumors return later, she says. A mouse model would also be better suited to follow tumor evolution than the faster larval fish version.

with that patient's tumor, we may be able to find something they respond to," he says.

Adult zebrafish are also headed towards the clinic. "Based on these studies, we have now opened a clinical trial for rhabdomyosarcoma using the same drugs identified using our zebrafish engrafment models," says Langenau.

Flies are flying ahead too, with trials currently underway at Mount Sinai for colorectal and medullary thyroid cancer patients who are not responding to traditional therapies. "We are making dozens and dozens of these [fly avatars]," says Cagan. "Each fly matches a different patient." For testing therapies, nearly half a million flies per line will be generated, and all 1500 FDA approved drugs screened in different combinations. The technology at work in New York is also being used by the company My Personal Therapeutics in the United Kingdom to offer fly avatars to clinicians and patients.

Finding the right combination of drugs will likely be key. "It's hard to find the one magic bullet," says Berman. "We're going to need combination drugs...but it's not just which drugs-it's which order do you give them in? Which comes first, which comes next? Do you give them the same time?"

Flies have a number advantage, but even the zebrafish offer greater scale and speed than a mouse model (Box 1). But is the future moving towards treating every patient as an $\mathrm{N}$ of 1 ?

"I don't know the answer to that," says Cagan. "I just want to see something work first, then let's work backwards and maybe we can learn some principles," he says.
In theory, one would want to use the fish to inform the mouse and then take a treatment to a patient, says Berman. But again, it can come down to time. "Sometimes decisions are being made on no data," says Berman, "It's, 'you have this disease, so let's just try this drug." Better to have an initial starting point than no in vivo information at all.

Novel combinations and new compounds will be discovered using fish or fly, says Langenau, but they will likely still need to make their way through mammalian xenograft testing to provide the highest preclinical rational for moving into clinical trials. "It is an exciting time for the field, with much to be learned and many models to leverage in our goal to identify new therapies for cancer," he says.

Many of the fly avatars share the same cancer genes, but they may not capture all of a patient's 'passenger mutations' that mayor many not-be influencing outcomes.. "We have treated patients with some really astounding combinations that I just have no idea why they would work, but in some cases they do," he says. "What we're hoping to find is that it won't be... a different cocktail for every single patient."

Cracking that code is still to come, but every avatar may help bring it a little bit closer.

\section{Ellen P. Neff}

Lab Animal.

e-mail:ellen.neff@us.nature.com

Published online: 24 March 2020

https://doi.org/10.1038/s41684-020-0511-7

References

1. Lee, L. M. J., Setfor, E. Z., Bonde, G., Cornell, R. A. \& Hendrix, M. J. C. Dev. Dyn 233, 1560-1570 (2005).

2. Corkery, D. P., Dellaire, G. \& Berman, J. N. Br J Haematol 153, 786-789 (2011).

3. Bentley, V. L. et al. Haematologica 100, 70-76 (2015).

4. Costa, B., Estrada, M.F., Mendes, R.V., Fior, R. Cells 9 https://doi, org/10.3390/cells9020293 (2020).

5. Fior, R. et al. Proc. Natl Acad. Sci. USA. 114, E8234-E8243 (2017).

6. Costa, B. et al. EBioMedicine 51, 102578 (2020).

7. White, R. M. et al. Cell Stem Cell 2, 183-189 (2008).

8. Yan, C. et al. Cell 177, 1903-1914 (2019).

9. Rajan, V. et al. Haematologica https://doi.org/10.3324/ haematol.2019.223040 (2019).

10. Villegas, S. N. Dis. Model. Mech. 12, dmm039032 (2019).

11. Cagan, R. L., Zon, L. I. \& White, R. M. Dev Cell 49, 317-324 (2019).

12. Vidal, M., Wells, S., Ryan, A. \& Cagan, R. Cancer Res. 65, 3538-3541 (2005).

13. Bangi, E., Murgia, C., Teague, A. G. S., Samson, O. J. \& Cagan, R. L. Nat. Commun. 7, 13615 (2016).

14. Bangi, E. et al. Sci Adv 5, eaav6528 (2019).

15. Ceol, C. J. et al. Nature 471, 513-517 (2011).

16. Hayward, N. et al. Nature 545, 175-180 (2017).

17. Callahan, S. J. et al. Dis. Model Mech. 11, dmm034561 (2018). 